\title{
Numerical study of a non-equilibrium interface model
}

\author{
Balakrishna Subramanian (sbala@physics.rutgers.edu) \\ Department of Physics, Rutgers University, Piscataway, NJ 08855, USA \\ G.T. Barkema (barkema@sns.ias.edu) \\ Institute for Advanced Study, Olden Lane, Princeton, NJ 08540, USA \\ J.L. Lebowitz*(lebowitz@math.rutgers.edu), E.R. Speer (speer@math.rutgers.edu) \\ Department of Mathematics, Rutgers University, New Brunswick, NJ 08903, USA
}

\begin{abstract}
We have carried out extensive computer simulations of one-dimensional models related to the low noise (solid-on-solid) non-equilibrium interface of a two dimensional anchored Toom model with unbiased and biased noise. For the unbiased case the computed fluctuations of the interface in this limit provide new numerical evidence for the logarithmic correction to the subnormal $L^{\frac{1}{2}}$ variance which was predicted by the dynamic renormalization group calculations on the modified Edwards-Wilkinson equation. In the biased case the simulations are in close quantitative agreement with the predictions of the Collective Variable Approximation (CVA), which gives the same $L^{\frac{2}{3}}$ behavior of the variance as the KPZ equation.
\end{abstract}

*Also Department of Physics, Rutgers University. 


\section{INTRODUCTION}

The nature of the interface separating two equilibrium phases, or more generally any two distinct bulk states of matter, is a problem of continuing interest. While there is in most cases some fuzziness in the transition region, giving rise to an intrinsic structure, the width of this is usually much smaller than (and therefore separable from) the fluctuations in the location of the interface. These fluctuations are fairly well understood both microscopically and macroscopically in (simple) equilibrium systems [1], but much less is known about them in the larger context of nonequilibrium situations [2]

An important advance in the latter case was the introduction of equations of the EdwardsWilkinson [3] and Kardar-Parisi-Zhang [4] type to describe fluctuations in dynamically moving interfaces. Solutions of these equations appear to describe in a quantitative manner the macroscopic fluctuations of a large class of such interfaces [2]. It is probably fair to say, however, that there are few microscopic systems (even simple ones) for which one can argue a priori, with mathematical rigor, that their behavior will be described by these equations.

Several years ago two of us (JLL and ES), together with B. Derrida and H. Spohn [5,6], introduced and studied the behavior of the low-noise-limit sharp interface separating the + and - phases in a simple model nonequilibrium system - the 2D Toom cellular automaton. Our semi-infinite interface was situated in the third quadrant of the square lattice and was anchored at the origin. Its precise location could be specified by a spin configuration on the positive semi-infinite one-dimensional integer lattice $\mathbf{Z}_{+}$. Fluctuations of the location of the interface at a distance $L$ from the origin are then directly transcribed into fluctuations of the magnetization $M_{L}=\sum_{i=1}^{L} \sigma_{i}$ in the stationary state of the one-dimensional model.

The dynamics of the semi-infinite 1D spin model are as follows: starting with some initial configuration (containing a mixture of + and - spins) we pick a site $i$ with rate 1 if $\sigma_{i}=-1$, and with rate $\lambda \leq 1$ if $\sigma_{i}=1$, and exchange $\sigma_{i}$ with the spin $\sigma_{j}$, where $j$ is the first site to the right of $i$ such that $\sigma_{j}=-\sigma_{i}$. Remarkably, the model inherits the unidirectional nature of information flow in the Toom model and the stationary state for the first $L$ spins can be 
obtained exactly (no finite size effects) by restricting the dynamics to a system with L spins, with the provision that if site $i$ is in the last block of spins, i.e., if $\sigma_{i}=\sigma_{i+1}=\ldots=\sigma_{L}$, then the spin $\sigma_{i}$ is flipped, i.e., changed to $-\sigma_{i}$ (with rate 1 or $\lambda$ ). The model can be classified into two cases, the unbiased case with $\lambda=1$, and the biased case with $\lambda \neq 1$.

Quantities of interest in the model include the average of the magnetization $\left\langle M_{L}\right\rangle$ and its variation $V_{L} \equiv\left\langle\left(M_{L}-\left\langle M_{L}\right\rangle\right)^{2}\right\rangle$ as functions of the system size $L$. By symmetry, $\left\langle M_{L}\right\rangle=0$ in the unbiased case, and a simple computation, using the well justified assumption that spins far from the origin are statistically independent, shows that in general $\left\langle M_{L}\right\rangle \simeq \mu L$ with $\mu=\frac{1-\sqrt{\lambda}}{1+\sqrt{\lambda}}$. The fluctuations have not been calculated exactly. Simulations reported in [5] gave $V_{L} \sim L^{\nu}$ with $\nu=0.53$ in the unbiased case and $\nu=0.57$ for $\lambda=1 / 4$, but in fact approximate treatments discussed there suggested that the true exponents are $\nu=1 / 2$ in the unbiased case, possibly with logarithmic corrections, and $\nu=2 / 3$ in the biased case.

Two approximation methods were introduced in [5]. The first, the collective variable approximation $(\mathrm{CVA})$, gives $V_{L}^{C V} \simeq \sqrt{3 / 2} L^{1 / 2}$ in the unbiased case and $V_{L}^{C V} \simeq C L^{2 / 3}$ (with $C$ a computable constant) in the biased case. The second approach was based on a description of the interface by a non-linear stochastic diffusion equation of the EW [3] and KPZ [4] type. Analysis of this equation predicts how fluctuations of a (doubly) infinite uniform interface will grow in time; in the semi-infinite problem, the fixed boundary at the left of the system and the positive velocity of excitations convert this growth to a corresponding $L$-dependence of $V_{L}$. This approach predicts $V_{L} \sim L^{2 / 3}$ in the biased case (here $2 / 3$ is the usual KPZ exponent). In the unbiased case the prediction depends on the growth of excitations in a modified KPZ equation with a third order, but no second order, nonlinearity; a subsequent renormalization group analysis of this problem ( [7], see also [8]) leads to the prediction $V_{L} \sim L^{1 / 2} \log ^{1 / 4}\left(L / L_{0}\right)$.

The simulation results of [5] did not include sufficiently large systems to distinguish the logarithmic behavior in the unbiased case from behavior $L^{\nu}$, with $\nu$ a power slightly higher than $1 / 2$, or to verify the power $2 / 3$ in the biased case. Simulations of [7] and [8] support the renormalization group conclusions for a uniform system but, since the passage from the 
uniform to the semi-infinite system is somewhat heuristic, provide only indirect guidance for the latter. In the present note we describe new simulations on our original model for $L \leq 2^{19}$ both for $\lambda=1$ and $\lambda=1 / 4$. We also did simulations on modified models and models with different bias values. Our results on the unbiased case give support to the predictions of [7]. Our results for the biased case seem to suggest that the CVA is a very good approximation.

For computational efficiency, we used a type of "multi-spin coding", similar to the technique applied for simulating the repton model in [9.10]. The computationally intensive part of the code is written in bit operations like AND $(\wedge)$, exclusive OR $(\oplus)$ and NOT $(\neg)$. This allows one to run 32 or 64 independent simulations in parallel by applying bit operations to four-byte or eight-byte integers of which each bit corresponds to an independent simulation.

We introduce a coding variable $\eta_{j}=\left(1+\sigma_{j}\right) / 2$. To implement the algorithm, we first choose (as described below) a marker $m$, whose nonzero bits select the simulations in which updating will take place, then pick a random site $j$ and flip the selected spins at this site:

$$
\eta_{j}^{\prime}=m \oplus \eta_{j}
$$

Next we walk along the chain, starting at $k=j+1$ and incrementing $k$ by one at each step. In each simulation we flip the spin $\sigma_{k}$ if it differs from $\sigma_{j}$ and if so we set the corresponding bit in the marker to zero:

$$
\begin{aligned}
d & =\eta_{k} \oplus \eta_{j} \\
\eta_{k}^{\prime} & =\eta_{k} \oplus(m \wedge d) \\
m & =m \wedge \neg d
\end{aligned}
$$

We continue walking until either our marker $m$ equals $\overrightarrow{0}$ or we have reached the end of the chain. To insure that simulations running in different bits do not become identical, we initialize $m$ as $m=r$, where $r$ is a random bit-sequence with each bit taking the value 1 with probability $1 / 2$, and then repeat with a different starting site $j$ and with $m=\neg r$. This generates a large degree of independence between simulations corresponding to different bits. 
To obtain the mask $m$ we use the random number generator marsag [11], which has good randomness properties for all the bits; for the site selection we use the random number generator ranmar [1], which has a long sequence and good spectral properties.

In the absence of a bias $(\lambda=1)$, the thermalization time $t_{0}$ required was determined to be $t_{0}=L^{2} / 8$ spin exchanges. The autocorrelation time of the magnetization in the steady state is much smaller, growing as $L^{3 / 2}$, as found also in [5], although the thermalization time suggests that some correlations must grow as $L^{2}$. The presence of a bias increases the required thermalization time; for $\lambda=1 / 4, t_{0} \approx L^{2} / 4$ and for $\lambda=1 / 8, t_{0} \approx L^{2} / 2$. For $\lambda=1 / 2$ we took again $t_{0} \approx L^{2} / 4$ as thermalization, although we could have taken a bit less. One run typically starts with a random spin configuration, which is evolved over time $(n+1) t_{0}$. The magnetization in each simulation (corresponding to a bit) is measured every $L$ moves; for each interval of length $t_{0}$ a separate histogram of the magnetization $\mathrm{M}$, including all bit simulations, is constructed. The first histogram is discarded (thermalization) and from the remaining $n$ histograms we obtain moments of the magnetization. Reported statistical errors in these moments are one standard deviation errors based on the assumption that these $n$ histograms are statistically independent. Note that even if simulations in different bits are correlated, we still obtain a reliable error estimation.

\section{THE UNBIASED CASE}

In the absence of bias $(\lambda=1)$ we now have data for sizes $L \leq 2^{19}$, presented in Table I. Two analytic descriptions of the growth of fluctuations have been proposed: in [5] it was suggested that:

$$
V_{L} \simeq C \cdot L^{\nu}
$$

with $\nu \simeq 0.5$; on the other hand in [7] (see also [8]), a formula based on the dynamical renormalization calculation was proposed:

$$
V_{L} \simeq C \cdot L^{1 / 2} \cdot \log ^{\beta}\left(L / L_{0}\right)
$$


with $\beta=1 / 4$ and $L_{0}=8$. In this section we will argue that this data supports the conclusion of [7], although we disagree on the identification of the constant $L_{0}$.

Clearly it is difficult to distinguish between the behavior (5) and (6). For example, the quantity $V_{L} /\left(L^{1 / 2} \log L\right)$ is a monotonic decreasing function of $L$ throughout the range of our simulations and $V_{L} /\left(L^{1 / 2} \log ^{1 / 8} L\right)$ is monotonic increasing for $L \geq 64$, suggesting asymptotic behavior (6) for some intermediate value of $\beta$, but in fact the same is true if $V_{L}$ is replaced by $L^{0.53}$, the behavior found in [5].

As a qualitative criterion for comparison of fit, we ask for what minimal value $2^{K}$ of the system size various proposed forms (all having two free parameters) can provide a good fit over interval $\left[2^{K}, 2^{19}\right]$, rejecting a fit as bad if it fails the standard $\chi^{2}$ test at a $99 \%$ confidence level. The asymptotic form (5), computed as a linear fit $\log \left(V_{L} / L^{1 / 2}\right) \sim a+b \log L$, yields $K=12$, while the forms (6), again as linear fits $\left(V_{L} / L^{1 / 2}\right)^{1 / \beta} \sim a_{\beta}+b_{\beta} \log L$, yield $K=11$, 6,8 , and 10 for $\beta=1,1 / 2,1 / 4,1 / 6$, respectively. This analysis thus provides evidence to prefer (6), which describes the data over a wider range of system sizes, although it does not conclusively rule out (5).

We now consider (6) and ask whether our data can determine the exponent $\beta$. The difficulty is that the three parameters in (6) provide a great deal of freedom, allowing for good fits for a range of values of $\beta$, if we vary the values of $L_{0}$ and $C$. The test discussed above is indefinite, and in fact mildly favors a value of $1 / 2$, as opposed to the renormalization group calculation of $1 / 4$. We now turn to a different line of argument which does provide evidence for the latter value, and begin with a review of the second approximate approach of [5].

As mentioned in the introduction, the application of the renormalization group depends on consideration of our dynamical model on the doubly infinite line, viewed as a height interface growth model. Such interface growth models may be described by non-linear stochastic diffusion equations of the EW and KPZ type; for our model the symmetries in the problem lead to the equation 


$$
\frac{d h}{d t}=\nu \frac{d^{2} h}{d x^{2}}+v_{1} \frac{d h}{d x}+v_{3}\left(\frac{d h}{d x}\right)^{3}+\eta(x, t)+\text { higher order terms }
$$

where $\eta$ is a stochastic noise term. The term in (17) with coefficient $v_{1}$ is usually eliminated by a Galilean transformation, but this is not possible in the semi-infinite system and in fact this term has important consequences, discussed below. If $v_{3}=0$ then (7) is the EdwardWilkinson growth model, which leads to interface growth $\left\langle h(t)^{2}\right\rangle \sim t^{\frac{1}{2}}$. The full equation (7) can be analyzed using dynamical renormalization techniques [7]; in this analysis the higher order terms are irrelevant and the cubic term is marginally irrelevant, giving

$$
\left\langle h(t)^{2}\right\rangle \simeq A t^{\frac{1}{2}} \log ^{\frac{1}{4}}\left(t / t_{0}\right)
$$

The parameter $v_{1}$ is positive, so that fluctuations in $h(t)$ travel to the right. We can compute the velocity $v$ of infinitesimal density perturbations in the (Bernoulli) steady state, via $v=\partial J /\left.\partial \rho\right|_{\rho=1 / 2}$, where $J(\rho)$ is the current at density $\rho$, to find $v=8[7]$. To apply these observations to the original model on the half open system it was suggested in [5] that excitations created at the closed end of the system grow according to (8) as they travel towards the open end at speed $v$; this leads to (6) with $\beta=1 / 4, C=A / v^{1 / 2}$, and $L_{0}=v t_{0}$. (See also [7], although there it is tacitly assumed that $t_{0}=1$ ).

In order to understand the exact form of the logarithmic corrections and check the validity of the above prediction, we studied a class of modifications of the original model, with dynamics defined as follows: we choose a site at rate 1, then exchange the spin at that site with the $k^{\text {th }}$ spin of opposite sign to the right. The original model is obtained by taking $k=1$. These models all share the symmetries of the original, so we expect that they will belong to the same universality class and hence have similar behavior of fluctuations; thus the variance $V_{L}(k)$ in these models should satisfy (6) with the same value of $\beta$ but with possibly different constants $L_{0}(k)$ and $C(k)$. We obtained data for models with $k=2,3$ (see Table I). Again, we can fit (6) to the individual data sets for a range of values of $\beta$ if we choose appropriate $L_{0}(k)$ and $C(k)$; to proceed further, we would like some a priori argument determining how these values depend on $k$. 
Now we propose a heuristic argument which describes how the constant $C(k)$ might vary with $k$. On the one hand, a computation in the steady state determines that the current in this model is proportional to $k$ and hence that the velocity $v(k)$ of excitations satisfies $v(k)=k \cdot v(1)=8 k$. On the other hand, the model for parameter $k$ can be thought of as a modification of the original model in which, during one time step, $k$ exchanges rather than one spin exchange take place. Now these $k$ exchanges take place in correlated positions. These correlations are certainly significant on the microscopic scale (e.g., the distance between two successive moves would definitely be affected by these correlations), but as seen from the computation of the current and the velocity these correlations are apparently not important on the hydrodynamic scale. Hence there should be some length scale below which the correlations are significant. We have two parameters in (6) which could be affected by these correlations, $C(k)$ and $L_{0}(k) . C(k)$ (along with $\beta$ ) determines the behavior to leading order at large $L$, and $L_{0}(k)$ is significant only at higher orders. If we assume that the correlations are not important at least to the order determining $C(k)$, then for a computation of $C(k)$, the model for parameter $k$ would correspond to the original model with the time rescaled by a factor of $k$. This would imply that $A(k) \sim k^{1 / 2} A(1)$, and hence that $C(k)=A(k) / v(k)^{1 / 2}=C(1)$.

Another way of saying this, which does not make any reference to the time dependent problem, is to write $\left\langle M_{L+1}^{2}\right\rangle=\left\langle M_{L}^{2}\right\rangle+2\left\langle\sigma_{L+1} M_{L}\right\rangle+1$. Since $\left\langle M_{L+1}^{2}\right\rangle-\left\langle M_{L}^{2}\right\rangle \rightarrow 0$ as $L \rightarrow \infty$, we must have $\left\langle\sigma_{L+1} M_{L}\right\rangle \rightarrow-\frac{1}{2}+o(L)$. Now if the leading order term in $o(L)$ is also independent of $k$ (which seems not unreasonable) then we would also have $C(k)$ independent of $k$ and vice versa.

To study the $k$-dependence of $C(k)$, we plot in Figure $1\left(V_{L}(k) / L^{1 / 2}\right)^{1 / \beta}$ versus $\log L$ for $\beta=1 / 2,1 / 4$, and $1 / 6$; according to equation (6) this curve is approaching a straight line with slope $C(k)^{1 / \beta}$ and offset $C(k)^{1 / \beta} \log \left(L_{0}\right)$. Only for $\beta=1 / 4$ can a data collapse be obtained by vertically shifting the curves (which corresponds to a change in $L_{0}$ ), indicating that $k$-independence of $C(k)$ holds only for $\beta=1 / 4$. Results of least-squares fit for all data on the interval $\left[2^{10}, 2^{18}\right]$, in the form $\left(V_{L} / L^{1 / 2}\right)^{1 / \beta} \sim a_{\beta}+b_{\beta} \log L$, are given in Table 2 ; the 
approximate constancy of $b_{1 / 4}(k)$ verifies the conclusion. Thus under the assumption made above, this provides independent justification of the conclusion $\beta=1 / 4$.

We also observe from Table 2 that $a_{\beta}(k)$ and hence $L_{0}(k)$ depends strongly on $k$ for all $\beta$. We interpret this as indicating that the correlations are important on the scale of $L_{0}$ and hence we cannot use the above argument to determine the $k$ dependence of $L_{0}(k)$.

To summarize, we now have numerical evidence for logarithmic corrections, and our data, when supplemented by a heuristic argument, supports the prediction (6) (with $\beta=1 / 4$ ) obtained with the help of dynamical renormalization techniques [7].

\section{THE BIASED CASE}

In the biased case we generated data for $L \leq 2^{18}$ for $\lambda=1 / 4$. As compared to the data reported in [5], this provides much better evidence for the asymptotic form $V_{L} \simeq B L^{2 / 3}$ predicted by both the KPZ and CVA approximations. We note that for the biased model the differential equation for the height evolution is the usual KPZ equation

$$
\frac{d h}{d t}=\nu \frac{d^{2} h}{d x^{2}}+v_{1} \frac{d h}{d x}+v_{2}\left(\frac{d h}{d x}\right)^{2}+\eta(x, t)+\text { higher order terms }
$$

where now the the non-linear quadratic term is relevant and it changes the power of the growth of fluctuations in time from $1 / 2$ to $2 / 3$.

We now compare the simulation results more closely with those obtained analytically for the asymptotic case of the CVA (the continuum limit): $B^{C V}=1.544 \lambda^{1 / 3}(1-\sqrt{\lambda})^{2 / 3}(1+$ $\sqrt{\lambda})^{-2}[5]$. Note that $B^{C V}$ vanishes at $\lambda=0,1$ and has a maximum at $\lambda=7-4 \sqrt{3} \approx 0.0718$. As shown in Figure 2, the values obtained from the CVA seem to be strikingly close to the simulation values in the asymptotic limit. The data suggests that the constants of proportionality $B$ for the variance in the two cases are extremely close if not identical. To check whether the CVA was close only for this particular value of $\lambda$, we studied two more values of bias $\lambda=1 / 2,1 / 8$. As shown in Figure 2 for both the cases, we observed that the CVA seemed to give values which are extremely close to the actual values. 
In order to have a better estimate of the accuracy of the CVA, we also studied the whole distribution of magnetization. The continuum limit CVA gives for the distribution of the magnetization values the fourth power of an Airy function [5], with a somewhat ad hoc cutoff suggested by the discrete CVA (the simulations and the CVA both give a Gaussian distribution for $\lambda=1$ ). This function has an asymmetry about the mean value. In Figure 3 we compare this CVA limiting distribution with the distribution of magnetization determined from simulations, with all distributions normalized to have a mean of 0 and a standard deviation of 1 . It can be seen that the actual distribution shows the same characteristic features as the CVA result; moreover, looking at the distributions for different L values suggests a slow approach of the distribution towards the Airy function with increasing size L. However, if we look at the leading measure of asymmetry in the distribution - the normalized third moment $\frac{\left\langle(M-\langle M\rangle)^{3}\right\rangle}{\left\langle(M-\langle M\rangle)^{2}\right\rangle^{3 / 2}}$ - then we observe (see Figure 3 inset) that the actual values and that obtained from the CVA are quite different.

We also studied the values of average magnetization obtained for finite size systems. As noted in [5] the exact asymptotic value and that obtained from the CVA coincide and are given by $\left\langle M_{L}\right\rangle=\mu L$, where $\mu=\frac{1-\sqrt{\lambda}}{1+\sqrt{\lambda}}$. In Figure 4 , we plot the approach of the average magnetization to the asymptotic value. Both the approach for the CVA and that for the actual data show very similar behavior.

At the end of this analysis, we are left with the somewhat puzzling result, that though the CVA does not reproduce, even qualitatively, the results for the unbiased case, it seems to be an extremely accurate description for the biased case.

\section{Acknowledgments}

We thank T. Hwa, G. Schütz and H. Spohn for useful discussion and communications. GTB acknowledges financial support from the DOE under grant DE-FG-90ER-40542, and from the Monell foundation. BS and JLL were supported in part by NSF Grant 9213424 . JLL would also like to thank DIMACS and its supporting agencies the NSF under contract STC-91-19999 and the N.J. Commission on Science and Technology. 


\section{REFERENCES}

[1] See articles by D.B. Abraham, H.W. Diehl and D. Jasnow in Phase Transitions and Critical Phenomena V. 10, eds. C. Domb and J.L. Lebowitz, New York, Academic Press, 1986.

[2] J. Krug, and H. Spohn, "Kinetic Roughening of Growing Surfaces," in Solids Far from Equilibrium, ed. C. Godreche, Cambridge University Press, 1991.

[3] S.F. Edwards and D.R. Wilkinson, Proc. R. Soc, London, Ser. A 381, 17 (1982).

[4] M. Kardar, G. Parisi and Y.C. Zhang, Phys. Rev. Lett. 56, 889 (1986).

[5] B. Derrida, J.L. Lebowitz, E.R. Speer and H. Spohn, J. Phys A. 24, 4805 (1991).

[6] B. Derrida, J.L. Lebowitz, E.R. Speer and H. Spohn, Phys. Rev. Lett. 67, 165 (1991).

[7] M. Barma, M. Pascuski, T. Hwa and S. Majumdar, Phys. Rev. Lett. 69, 2735 (1992).

[8] P. Devillard and H. Spohn, J. Stat. Phys. 66, 1089 (1992).

[9] G.T. Barkema, J.F. Marko, and B. Widom, Phys. Rev. E 49, 5303 (1994).

[10] G.T. Barkema and G.M. Schütz, Europhys. Lett.35, 139 (1996).

[11] G. Marsaglia, Private Communication. 


\section{FIGURES}

FIG. 1. If $V_{L} \approx C(k) L^{1 / 2} \log ^{\beta}\left(L / L_{0}\right)$, then $\left(V_{L} / L^{1 / 2}\right)^{1 / \beta}$ as a function of $\log L$ is a straight line with slope $C(k)^{1 / \beta}$. In the figure, $\left(V_{L} / L^{1 / 2}\right)^{1 / \beta}$ is plotted as a function of $\log L$ for $k=1$ (plusses), $k=2$ (circles), and $k=3$ (diamonds), and $\beta=1 / 4$. The curves for $k=1$ and $k=2$ are shifted vertically, to obtain a collapse. The insets show that for $\beta=1 / 2$ (left inset) and $\beta=1 / 6$ (right inset) the data does not collapse.

FIG. 2. $V_{L} / L^{2 / 3}$ is plotted as a function of $\log L$, for $\lambda=1 / 8$ (diamonds), $\lambda=1 / 4$ (circles), and $\lambda=1 / 2$ (squares). In the first two cases the data shows convergence to a constant, indicating that $V_{L} \sim L^{2 / 3}$ without logarithmic corrections. The lines are the CVA values for the corresponding biases; the asymptotic CVA values are $0.3150,0.2723$ and 0.1855 , respectively.

FIG. 3. Normalized distribution (with mean 0 and standard deviation 1) of the magnetization $M_{L}$ for the biased case $(\lambda=1 / 4)$ with system sizes $L=1024$ (dashed line) and $L=262144$ (dotted line) together with the normalized distribution obtained from the asymptotic CVA (solid line). The inset shows the third moment of the magnetization, as a function of $\log L$, for the CVA (solid line) and for the simulation(circles).

FIG. 4. Finite-size effects in the magnetization for $\lambda=1 / 4$ : the deviation from $\left\langle M_{L}\right\rangle / L=1 / 3$ versus system size $L$ is given in a log-log plot. The solid line is the CVA, the circles are simulation results. 


\section{TABLES}

\begin{tabular}{rccc}
\hline \hline$L$ & $V_{L}=V_{L}(1)$ & $V_{L}(2)$ & $V_{L}(3)$ \\
\hline 2 & $1.335[1]$ & 2 & 2 \\
4 & $1.918[1]$ & $2.75[1]$ & $3.637[1]$ \\
8 & $2.733[1]$ & $3.818[1]$ & $4.626[2]$ \\
16 & $3.890[1]$ & $5.271[1]$ & $6.451[2]$ \\
32 & $5.576[2]$ & $7.338[3]$ & $8.868[6]$ \\
64 & $8.02[2]$ & $10.286[4]$ & $12.292[6]$ \\
128 & $11.58[3]$ & $14.543[4]$ & $17.20[1]$ \\
256 & $16.76[3]$ & $20.66[1]$ & $24.27[2]$ \\
512 & $24.22[4]$ & $29.44[2]$ & $34.37[2]$ \\
1024 & $35.01[3]$ & $41.96[6]$ & $48.70[3]$ \\
2048 & $50.54[9]$ & $59.94[8]$ & $69.20[3]$ \\
4096 & $73.1[1]$ & $85.6[2]$ & $98.41[4]$ \\
8192 & $105.3[2]$ & $122.4[1]$ & $139.90[7]$ \\
16384 & $151.7[4]$ & $174.6[3]$ & $199.2[2]$ \\
32768 & $218.3[5]$ & $249.9[4]$ & $283.1[3]$ \\
65536 & $313[1]$ & $355.4[4]$ & $402.4[5]$ \\
131072 & $449[2]$ & $507[3]$ & $573[2]$ \\
262144 & $644[4]$ & $720[2]$ & $813[3]$ \\
524288 & $924[3]$ & & $1162[5]$ \\
\hline \hline
\end{tabular}

TABLE I. The variances obtained for the unbiased case for different values of $k$. The quantity in brackets after each value is the statistical error in the least significant digit. 


\begin{tabular}{lccr}
\hline \hline$k$ & $a_{1 / 2}$ & $a_{1 / 4}$ & $a_{1 / 6}$ \\
\hline 1 & $0.694 \pm 0.018$ & $0.086 \pm 0.050$ & $-0.969 \pm 0.102$ \\
2 & $1.376 \pm 0.023$ & $1.682 \pm 0.084$ & $1.551 \pm 0.228$ \\
3 & $2.052 \pm 0.018$ & $4.104 \pm 0.084$ & $7.924 \pm 0.300$ \\
\hline \hline$k$ & $b_{1 / 2}$ & $b_{1 / 4}$ & $b_{1 / 6}$ \\
\hline 1 & $0.073 \pm 0.002$ & $0.194 \pm 0.006$ & $0.386 \pm 0.013$ \\
2 & $0.050 \pm 0.003$ & $0.184 \pm 0.009$ & $0.507 \pm 0.025$ \\
3 & $0.038 \pm 0.002$ & $0.179 \pm 0.010$ & $0.640 \pm 0.036$ \\
\hline \hline
\end{tabular}

TABLE II. Values of $a_{\beta}$ and $b_{\beta}$ obtained from least-squares fits on the data for the unbiased model. 


\begin{tabular}{rccccc}
\hline \hline$L$ & $V_{L}$ & $\langle M\rangle_{L}$ & $\left\langle(M-<M>)^{3}\right\rangle_{L}$ & $V_{L}$ & $V_{L}$ \\
& $\lambda=\frac{1}{4}$ & $\lambda=\frac{1}{4}$ & $\lambda=\frac{1}{4}$ & $\lambda=\frac{1}{2}$ & $\lambda=\frac{1}{8}$ \\
\hline 1024 & $36.82[6]$ & $344.57[2]$ & $-0.171[6]$ & $35.7[1]$ & $37.0[2]$ \\
2048 & $55.3[1]$ & $686.98[2]$ & $-0.185[2]$ & $52.4[2]$ & $57.2[3]$ \\
4096 & $83.4[2]$ & $1371.06[3]$ & $-0.204[4]$ & $76.4[4]$ & $88.5[6]$ \\
8192 & $128.8[4]$ & $2738.32[5]$ & $-0.225[3]$ & $111.9[5]$ & $136.6[8]$ \\
16384 & $193[2]$ & $5471.4[1]$ & $-0.240[3]$ & $165.5[6]$ & $215[2]$ \\
32768 & $302.7[6]$ & $10936.1[3]$ & $-0.256[2]$ & $247[1]$ & $344[3]$ \\
65536 & $473[1]$ & $21862.62[6]$ & $-0.266[4]$ & $370[5]$ & $533[5]$ \\
131072 & $735[4]$ & $43713.2[2]$ & $-0.281[6]$ & $566[5]$ & $849[10]$ \\
262144 & $1165[7]$ & $84105.9[2]$ & $-0.266[5]$ & & \\
\hline \hline
\end{tabular}

TABLE III. The variances for three different values of bias, and the first and third moments for $\lambda=\frac{1}{4}$. Note that we have not obtained data for systems of size smaller than 1024 . The statistical error in the least significant digit is given by the quantity in brackets. 
Fig: 1

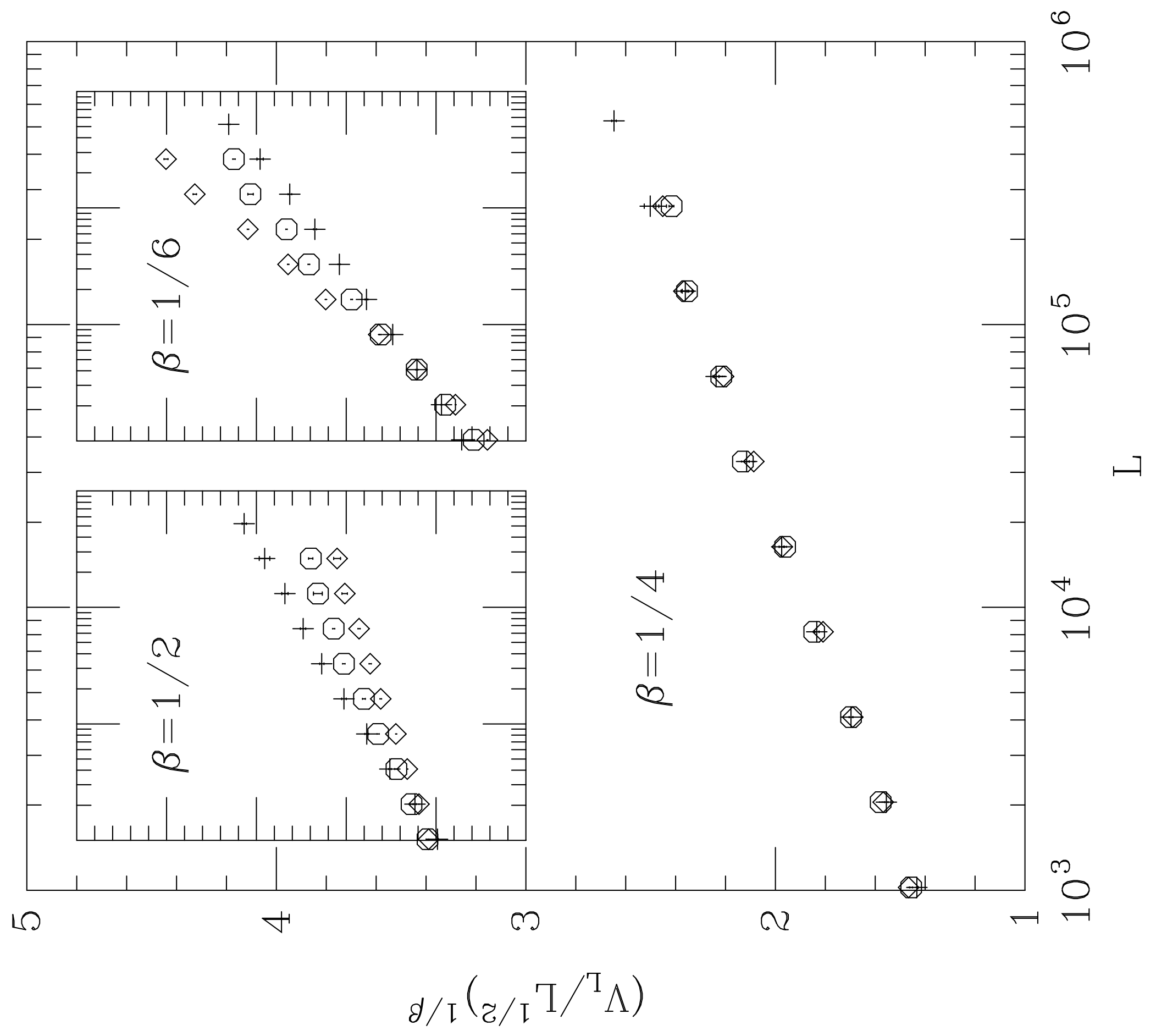


Fig: 2

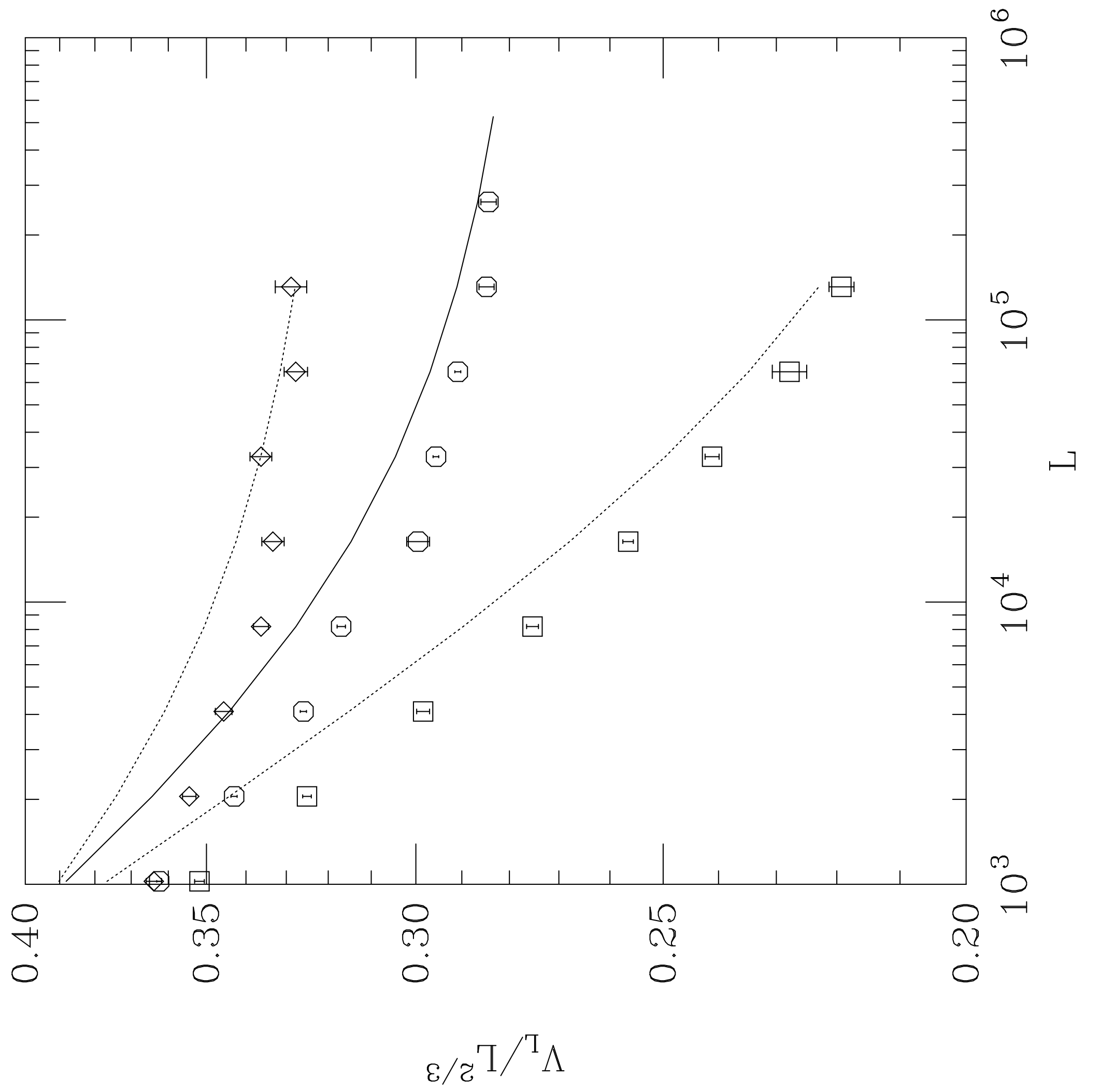


Fig: 3

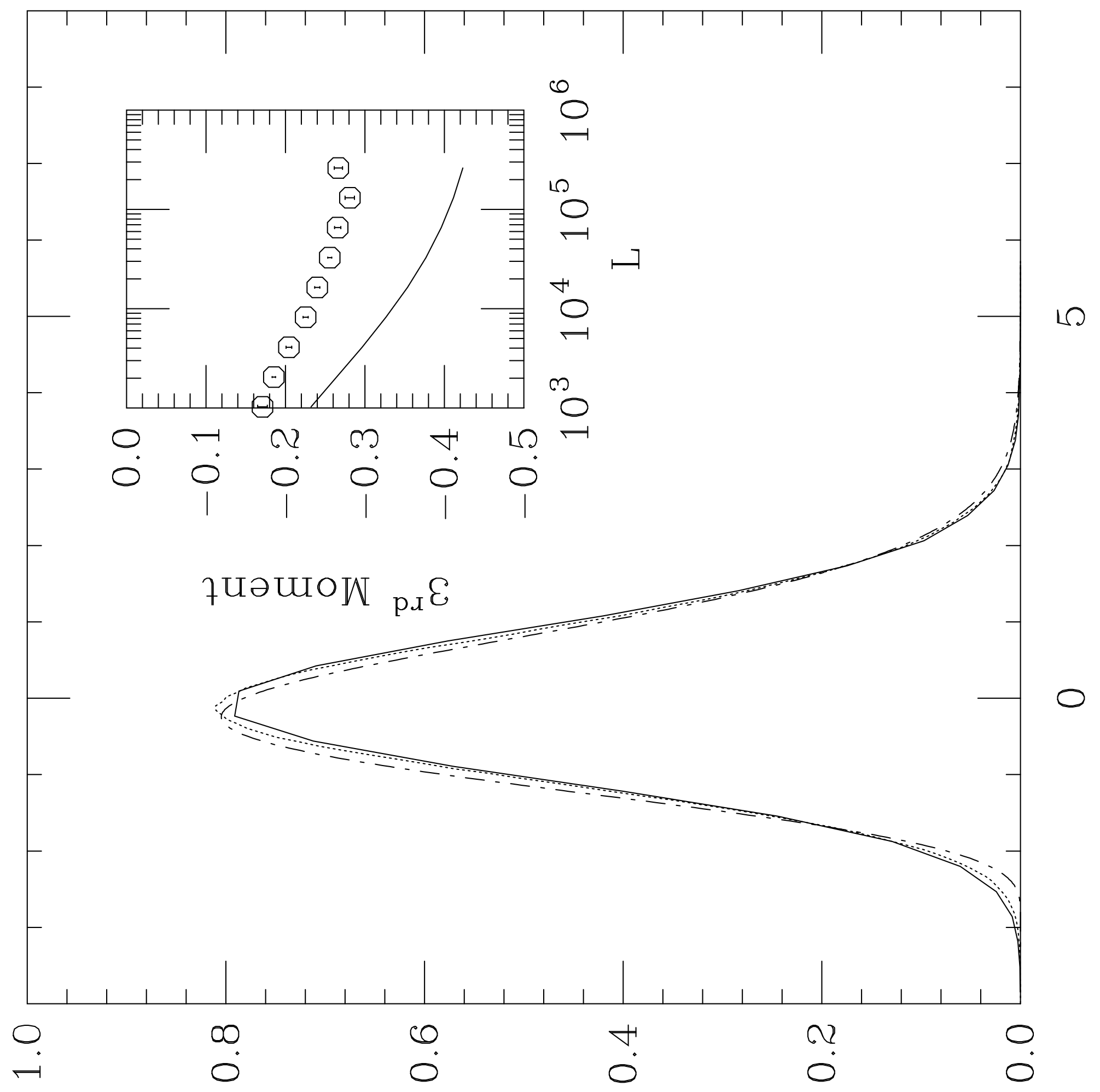


